\title{
Early Dabigatran Treatment After Transient Ischemic Attack and Minor Ischemic Stroke Does Not Result in Hemorrhagic Transformation
}

\author{
Anas Alrohimi (D), Kelvin Ng, Dar Dowlatshahi, Brian Buck, Grant Stotts (D), \\ Sibi Thirunavukkarasu, Michel Shamy (1), Hayrapet Kalashyan, Leka Sivakumar, \\ Ashfaq Shuaib, Mike Sharma, Ken Butcher (i)
}

\begin{abstract}
Objectives: The optimal timing of anticoagulation after ischemic stroke in atrial fibrillation (AF) patients is unknown. Our aim was to demonstrate the feasibility and safety of initiating dabigatran therapy within 14 days of transient ischemic attack (TIA) or minor stroke in AF patients. Patients and Methods: A prospective, multi-center registry (NCT02415855) in patients with AF treated with dabigatran within 14 days of acute ischemic stroke/TIA (National Institutes of Health Stroke Scale (NIHSS) $\leq 3$ ) onset. Baseline and follow-up computed tomography (CT) scans were assessed for hemorrhagic transformation (HT) and graded by using European Cooperative Acute Stroke Study criteria. Results: One hundred and one patients, with a mean age of $72.4 \pm 11.5$ years, were enrolled. Median infarct volume was $0 \mathrm{ml}$. Median time from index event onset to dabigatran initiation was 2 days, and median baseline NIHSS was 1. Pre-treatment HT was present in seven patients. No patients developed symptomatic HT. On the day 7 CT scan, HT was present in six patients (one progressing from baseline hemorrhagic infarction type 1). Infarct volume was a predictor of incident HT (odds ratio $=1.063$ [1.020-1.107], $p<0.003)$. All six $(100 \%)$ patients with new/progressive HT were functionally independent (modified Rankin Scale $(\mathrm{mRS})=0-2)$ at 30 days, which was similar to those without HT $(90 \%, p=0.422)$. Recurrent ischemic events occurred within 30 days in four patients, two of which were associated with severe disability and death (mRS 5 and 6, respectively). Conclusion: Early dabigatran treatment did not precipitate symptomatic HT after minor stroke. Asymptomatic HT was associated with larger baseline infarct volumes. Early recurrent ischemic events may be clinically more important.
\end{abstract}

RÉSUMÉ : Un traitement précoce au dabigatran à la suite d'un accident ischémique transitoire et d'un accident ischémique mineur n'entraîne pas de transformation de nature hémorragique. Objectifs: Le moment optimal pour entreprendre un traitement anticoagulant à la suite d'un accident ischémique demeure inconnu dans le cas de patients atteints de fibrillation auriculaire (FA). Nous entendons ici démontrer la faisabilité et la sécurité d'un traitement au dabigatran dans un délai de 14 jours après que des patients atteints de FA ont été victimes d'un accident ischémique transitoire (AIT) ou d'un accident ischémique mineur. Patients et méthodes: Il s'agit ici d'une étude prospective reposant sur les dossiers de patients traités dans plusieurs établissements (NCT02415855). On l'a dit, ces derniers étaient atteints de FA et ont été traités avec du dabigatran dans un délai de 14 jours suivant les premiers signes d'un accident ischémique aigu ou d'un AIT (score au NIHSS $\leq 3$ ). Des résultats à des examens de tomodensitométrie (TDM) de référence et de suivi ont été ensuite évalués pour détecter une transformation hémorragique (TH) et classés selon les critères de la European Cooperative Acute Stroke Study (ECASS). Résultats: Au total, nous avons inclus dans cette étude 101 patients dont l'âge moyen était de 72,4 $\pm 11,5$ ans. Le volume médian de leur infarctus était de $0 \mathrm{ml}$. Le délai médian entre les premiers signes d'un accident ischémique et les débuts d'un traitement au dabigatran a été de 2 jours tandis que le score médian au NIHSS au début de ce même traitement était de 1 . Si des TH prétraitement ont été observées chez 7 patients, il faut préciser qu'aucun d'entre eux n'a développé des TH de nature symptomatique. Lors des examens de TDM effectués au septième jour, des TH se sont révélées présentes chez 6 patients, dont un ayant évolué d'une absence de saignement à des écoulements de type 1 (HI1). Le volume des infarctus s'est quant à lui avéré un prédicteur de l'amorce d'une TH (rapport des cotes = 1,063 [1,020 1,107], $p<0,003$ ). Tous les 6 patients atteints de TH (nouvelles ou progressive) étaient considérés autonomes sur le plan fonctionnel (échelle modifiée de Rankin $=0-2)$ au bout de 30 jours, résultat similaire dans le cas de ceux n'étant pas atteints de TH $(90 \%, p=0,422)$. Enfin, notons que des évènements ischémiques récurrents se sont produits dans un délai de 30 jours chez 4 patients ; chez deux d'entre eux, cela a été associé respectivement à un grave handicap et à un décès (respectivement 5 et 6 à l'échelle modifiée de Rankin). Conclusions: Des traitements précoces au dabigatran n'ont pas entraîné des TH symptomatiques chez des patients victimes d'accidents ischémiques mineurs. Des TH de nature asymptomatique ont par ailleurs été associées à des volumes d'infarctus plus importants au début. Il est donc possible que des évènements ischémiques récurrents survenus de façon précoce soient plus importants d'un point de vue clinique.

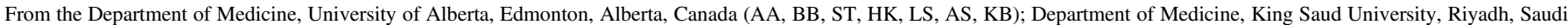

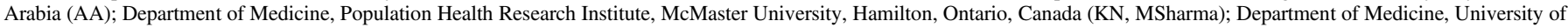
Ottawa, Ottawa, Ontario, Canada (DD, GS, MS); and Prince of Wales Clinical School, University of New South Wales, Sydney, NSW, Australia (KB)

Received January 12, 2020. Final Revisions Submitted February 29, 2020. Date of Acceptance April 18, 2020.

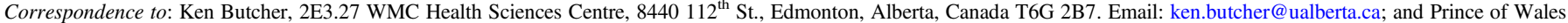

Clinical School, Level 1, South Wing, Edmund Blacket Building, Prince of Wales Hospital, Randwick, NSW 2031, Australia. Email: ken.butcher@unsw.edu.au 
Keywords: Atrial fibrillation, Ischemic stroke, Hemorrhagic transformation, Dabigatran, Cardioembolic stroke, Intracerebral hemorrhage

\section{INTRODUCTION}

Patients with atrial fibrillation (AF) who have suffered a transient ischemic attack (TIA) or ischemic stroke are at high risk for recurrence and require long-term anticoagulation. The risk of recurrent stroke (3-20\%) within the first two weeks after stroke/ TIA is clinically important. ${ }^{1,2}$ The optimal timing of anticoagulation after an ischemic stroke, however, remains controversial. Previous trials have demonstrated that anticoagulation in acute stroke patients is associated with reduced early stroke recurrence rates, but these benefits are offset by a comparable increase in the rate of symptomatic hemorrhagic transformation (HT). ${ }^{3,4}$ An individual patient-level meta-analysis reported that the hemorrhagic complication rate associated with low-molecular-weight heparin within 14 days of ischemic stroke was $0.8 \% .^{5}$ Similarly, early warfarin use in stroke patients without AF is associated with a significant increase in the rate of HT (relative risk 1.93, 95\% confidence interval 1.27-2.94). ${ }^{6}$

Dabigatran is a direct oral anticoagulant (DOAC), approved for the prevention of ischemic stroke in patients with AF. Dabigatran is associated with a lower risk of intracranial hemorrhagic complications than warfarin in patients with chronic AF without recent stroke. ${ }^{7}$ There are limited data related to the use of dabigatran within 14 days of stroke. All safety data related to dabigatran come from trials that excluded patients within 14 days of stroke and 3 days of a TIA. This is precisely the period when stroke patients are at highest risk of recurrent events and most likely to derive benefit from anticoagulation. Based on the limited data, Canadian Stroke Best Practice guidelines recommend basing the timing of anticoagulation on clinical severity and infarct size, but neither are well defined. ${ }^{8}$ This recommendation is based on expert consensus originally proposed in the European Heart Rhythm Association guidelines, and is not based on randomized clinical trial advice. ${ }^{9}$ The question of optimal timing of oral anticoagulation after ischemic stroke is frequently encountered in clinical practice. The guidance in this matter has been based largely on expert opinion and clinical experience, along with serial imaging.

\section{AIM}

The overall aim of this study was to demonstrate the feasibility and safety of initiating dabigatran therapy within 14 days of TIA or minor ischemic stroke in AF patients. We systematically assessed prospectively collected computed tomography (CT) scan images for evidence of HT.

\section{Methods}

The Canadian Pradaxa Acute Stroke Safety study was an investigator-initiated prospective, multicenter, open-label, singlearm phase IV study (clinicaltrials.gov registration NCT02415855). Patients with documented non-valvular AF (newly or previously diagnosed) with acute TIA (defined as acute focal neurological deficits, with complete resolution of symptoms within $24 \mathrm{~h}$ of onset) or mild ischemic stroke (National Institutes of Health Stroke Scale (NIHSS) score $\leq 3$ ) were enrolled. Study investigators approached patients after the treating physician's decision to treat with dabigatran within 14 days of stroke/TIA, independent of the registry. Informed consent was obtained from the patient or substitute decision maker in all cases prior to enrollment. The research protocol was approved by our local Human Research Ethics Board.

Patients were excluded if they had acute or chronic renal failure (estimated glomerular filtration rate (eGFR) $<30 \mathrm{ml} / \mathrm{min}$ ), known hypersensitivity to dabigatran or potential medical interactions with dabigatran therapy, and/or significant ongoing systemic bleeding risk.

\section{Procedures}

\section{Dabigatran Therapy}

The dabigatran dose was determined by the treating physician, based on age and renal function. Patients with an eGFR $30-50 \mathrm{ml} / \mathrm{min}$ and/or age $\geq 80$ years received $110 \mathrm{mg}$ twice a day. All other patients received $150 \mathrm{mg}$ twice a day.

\section{Clinical Assessments}

All study participants were followed for 30 days after dabigatran initiation. An NIHSS score was assessed, by certified study personnel at baseline, 7 and 30 days after enrollment. Functional outcome was assessed with a modified Rankin Scale (mRS) at baseline, 7, and 30 days. Montreal Cognitive Assessments were performed at baseline, day 7, and day 30, and quality of life was assessed with the EuroQol-5 Dimension (EQ-5D) and Visual Analog Scale. The Charlson Co-morbidity Index was calculated at baseline.

\section{Imaging Procedures and Analysis}

All patients had a non-contrast CT scan at baseline (within $24 \mathrm{~h}$ from study recruitment) and at $7 \pm 2$ days after enrollment using a non-contrast CT scan. In the event of clinical deterioration, CT scans were repeated.

Anonymized dicom CT data were analyzed centrally. Baseline and recurrent infarct volumes were measured using planimetric techniques (Analyze 11.0, Biomedical Imaging Resource, Biodynamics Research Unit, Mayo Foundation, Rochester, MN, USA). ${ }^{10}$ Any HT seen at baseline and day 7 was graded using European Cooperative Acute Stroke Study criteria: hemorrhagic infarction type 1 (HI1; small petechiae along the margins of the infarct), hemorrhagic infarction type 2 (HI2; confluent petechiae within the infarcted area but no space-occupying effect), parenchymal hemorrhage type 1 (PH1; blood clots in $30 \%$ or less of the infarcted area with some slight space-occupying effect), or parenchymal hemorrhage type 2 ( $\mathrm{PH} 2$; blood clot in more than $30 \%$ of the infarcted area with substantial space-occupying effect). ${ }^{11}$ 


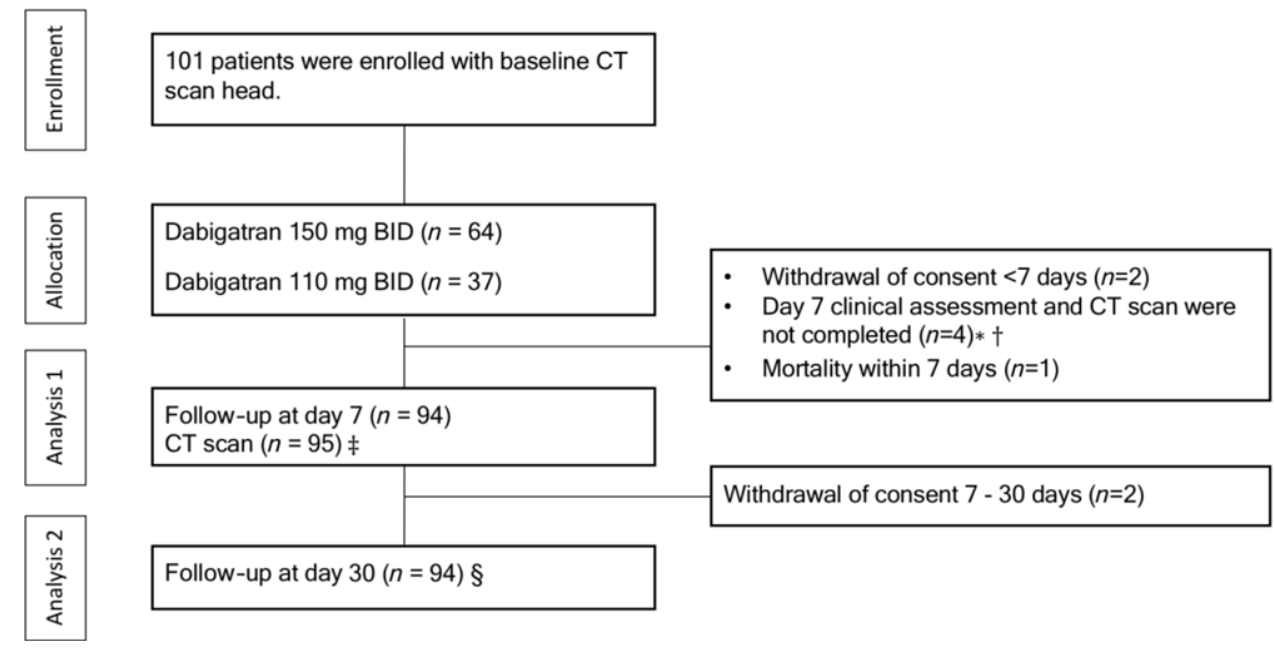

Figure 1: Consort diagram of enrollment, allocation, follow-up, and outcomes. The intention-to-treat population included all the patients who were enrolled in this study. All study participants were followed for 30 days after dabigatran initiation. Day 7 follow-up included clinical assessment and CT scan, while day 30 included clinical assessment. *One patient was in a rehabilitation hospital. ${ }^{*}$ Three patients were discharged to rural communities, without access to $C T$. ${ }^{\ddagger}$ One patient died within 7 days of enrollment as a consequence of recurrent ischemic stroke and concomitant systemic emboli, CT scan was repeated and included in the analysis. ${ }^{\S}$ Four patients whom clinical assessment and CT scan were not completed at day 7 follow-up, returned for their day 30 follow- up.

\section{Endpoints}

The primary endpoint was symptomatic HT, defined as $\mathrm{PH} 2$ associated with $\mathrm{a} \geq$ four-point increase in NIHSS score within 30 days of initiating dabigatran therapy. Secondary outcomes included any HT at day 7, systemic hemorrhagic complications, and recurrent ischemic stroke within 30 days of enrolment. Serious adverse events (SAEs) within the study period were recorded using standardized event, resolution and association codes, and reported to the local Human Research Ethics Boards and Health Canada.

\section{Sample Size and Statistical Analysis}

The study was an open-label registry not powered to demonstrate safety or efficacy. A sample of 100 patients was planned in order to obtain initial estimates of the frequency of symptomatic and asymptomatic HT. Given the lack of published data with respect to dabigatran in this population, the maximum acceptable rate of symptomatic HT was considered $2 \%$. This was based on a meta-analysis of low-molecular-weight heparin treatment in acute stroke, indicating the absolute symptomatic HT rate ranged from $2.4 \%$ to $2.9 \%{ }^{12}$ An a priori stopping rule therefore governed study continuation; enrollment would be halted immediately and permanently if $\geq 2$ symptomatic HT events occurred.

All statistical analyses were performed using the Statistical Package for Social Sciences version 23.0.0 (IBM SPSS Statistics Inc., 2015, Armonk, NY, USA). Differences between groups were assessed using independent $t$-tests for parametric data and Mann-Whitney $U$-tests for non-parametric data. Pearson's correlation coefficients were used to estimate the relationship between time to dabigatran initiation and infarct volume. The five-digit health state in EQ-5D was calculated using preference weights, as described previously. ${ }^{13}$

\section{Role of the Funding Source}

The trial was an Investigator-Initiated Study, funded by Boehringer-Ingelheim. The study sponsor was "The Governors of the University of Alberta." The protocol was written by the Principal Investigator (KB) and reviewed by the Boehringer-Ingelheim global medical team prior to funding. Boehringer-Ingelheim had no role in study design, data collection, analysis, interpretation, or manuscript preparation. Additional support was provided by the Canada Research Chairs Program and the Heart and Stroke Foundation of Alberta, Northwest Territories and Nunavut. The authors had full access to all data in the study and had final responsibility for the presentation of results.

\section{RESULTS}

\section{Baseline Characteristics}

Between December 2013 and February 2018, a total of 101 patients $(65 \%$ male) were enrolled at three Canadian stroke centers (Figure 1). Patient characteristics are summarized in Table 1. At the time of treatment initiation, median (interquartile range (IQR)) NIHSS $1(0-2)$ and the median (IQR) infarct volume was $0(0-7.43) \mathrm{ml}$.

The median (IQR) time from onset to first dabigatran dose was 2 (1-5) days for all patients (Figure 2). The majority of patients (64\%) received dabigatran $150 \mathrm{mg}$ twice a day, and the remainder received $110 \mathrm{mg}$ twice a day. The number of days between symptom onset and dabigatran initiation was directly correlated with the infarct volume $(r=0.49, p<0.0001)$. An acute infarct was visible on CT in 48 (48\%) of patients, and the most common location was cortical (35\%). Evidence of previous infarction was seen on baseline CT in 30 (30\%) patients. 
Table 1: Characteristics of patients with/without baseline HT

\begin{tabular}{|c|c|c|c|c|c|}
\hline & All patients $(n=101)$ & $\begin{array}{l}\text { Patients with baseline } \\
\text { HT }(n=7)\end{array}$ & $\begin{array}{c}\text { Patients without } \\
\text { baseline HT }(n=94)\end{array}$ & $\begin{array}{l}\text { Patients with incident } \\
\text { HT }(n=6)\end{array}$ & $\begin{array}{l}\text { Patients with recurrent } \\
\text { ischemic events }(n=4)\end{array}$ \\
\hline Mean \pm SD age (years) & $72.4 \pm 11.5$ & $71.6 \pm 9.3$ & $72.4 \pm 11.7$ & $70 \pm 5$ & $78.8 \pm 14.2$ \\
\hline Male, $n(\%)$ & $65(65 \%)$ & $5(71 \%)$ & $60(65 \%)$ & $4(67 \%)$ & $2(50 \%)$ \\
\hline CHA2DS2-VASc score, median (IQR) & $5(3-6)$ & $4(3.5-4.5)$ & $5(3-6)$ & $4(3-6)$ & $4.5(3.5-5.5)$ \\
\hline HAS-BLED score, median (IQR) & $2(2-3)$ & $2(2-3.5)$ & $2(2-3)$ & $2.5(2-3)$ & $3(2.5-3.5)$ \\
\hline Infarct volume, ml, median (IQR) & $0(0-7.43)$ & $31.2(27.8-47.5)$ & $0(0-5.1)$ & $27.3(13-48.5)$ & $2(1.5-3.5)$ \\
\hline \multicolumn{6}{|l|}{ Infarct location } \\
\hline Cortical, $n(\%)$ & $35(35 \%)$ & $5(71 \%)$ & $30(32 \%)$ & $6(100 \%)$ & $3(75 \%)$ \\
\hline Subcortical, $n(\%)$ & $10(10 \%)$ & $2(29 \%)$ & $8(9 \%)$ & $0(0 \%)$ & $1(25 \%)$ \\
\hline Posterior fossa, $n(\%)$ & $3(3 \%)$ & $0(0 \%)$ & $3(3 \%)$ & $0(0 \%)$ & $0(0 \% 0$ \\
\hline No lesion, $n(\%)$ & $53(52 \%)$ & $0(0 \%)$ & $53(56 \%)$ & $0(0 \%)$ & $0(0 \%)$ \\
\hline Baseline NIHSS, median (IQR) & $1(0-2)$ & $2(1-2)$ & $1(0-2)$ & $1(1-2)$ & $2(1-3)$ \\
\hline Day 30 NIHSS, median (IQR) & $0(0-1)$ & $1(0.5-1)$ & $0(0-1)$ & $0(0-1)$ & $0(0-8)$ \\
\hline Baseline mRS, median (IQR) & $0(0-0)$ & $1(0-2)$ & $0(0-0)$ & $0(0-0)$ & $0.5(0-1.5)$ \\
\hline Day 30 mRS, median (IQR) & $1(0-2)$ & $2(1-2)$ & $1(0-2)$ & $2(2-2)$ & $3.5(1.5-5.5)$ \\
\hline $\begin{array}{l}\text { Baseline Charlson Co-morbidity Index, } \\
\text { median (IQR) }\end{array}$ & $4(2-5)$ & $3(3-4.5)$ & $4(2-5)$ & $3(3-4)$ & $5.5(3.5-6)$ \\
\hline Baseline MoCA, median (IQR) & $24.5(21.25-26)$ & $24.5(21-26)$ & $24.5(21-26)$ & $23(22-24)$ & $24.5(19-26)$ \\
\hline Day 30 MoCA, median (IQR) & $25(23-27.5)$ & $24.5(21-28)$ & $25(23-27)$ & $27(26-28)$ & $25(23-27.5)$ \\
\hline Mean (SD) EQ-5D index score at day 30 & $0.876 \pm 0.11$ & $0.91 \pm 0.11$ & $0.87 \pm 0.11$ & $0.84 \pm 0.01$ & $0.82 \pm 0.01$ \\
\hline $\begin{array}{l}\text { Mean (SD) EQ-5D Visual Analog Scale } \\
\text { score at day } 30\end{array}$ & $71.8 \pm 18.3$ & $69.75 \pm 24.6$ & $72 \pm 18.1$ & $75 \pm 7$ & $72.5 \pm 3.5$ \\
\hline $\begin{array}{l}\text { Time to dabigatran initiation (days), } \\
\text { median (IQR) }\end{array}$ & $2(1-5)$ & $5(4.5-9)$ & $2(1-5)$ & $6(2-9)$ & $2(1.5-3.5)$ \\
\hline
\end{tabular}

CHA2DS2-VASc Score, congestive heart failure, hypertension, age, diabetes mellitus, stroke (doubled), vascular disease, age, and sex category (female); HAS-BLED Score, hypertension, abnormal renal/ liver function, stroke, bleeding history or predisposition, labile international normalized ratio, elderly, drugs/alcohol concomitantly; MoCA, Montreal Cognitive Assessment.

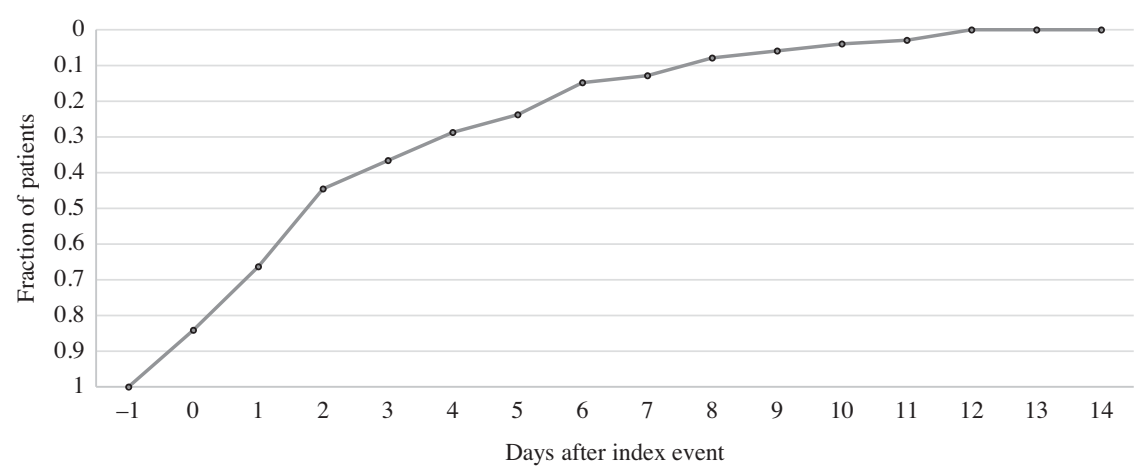

Figure 2: Time from ischemic stroke/TIA onset to dabigatran initiation in all patients. Patients initiated on day 0 received their first dose on the day of the index event. The median time to dabigatran initiation was 2 days.

\section{Dabigatran Compliance and Follow-up}

During the follow-up period, two patients withdrew consent prior to the follow-up CT scan at day 7. Two patients withdrew consent after the day 7 assessment. One patient died within 7 days of enrollment as a consequence of recurrent ischemic stroke and concomitant systemic emboli. A CT scan was not obtained in four patients at day 7. A follow-up CT scan was obtained in 95 patients at a median (IQR) of 7 (5-8.5) days after dabigatran initiation. One patient was non-compliant on dabigatran therapy at day 7 and developed a recurrent ischemic event. The remaining patients were compliant at day 7 and 30. 


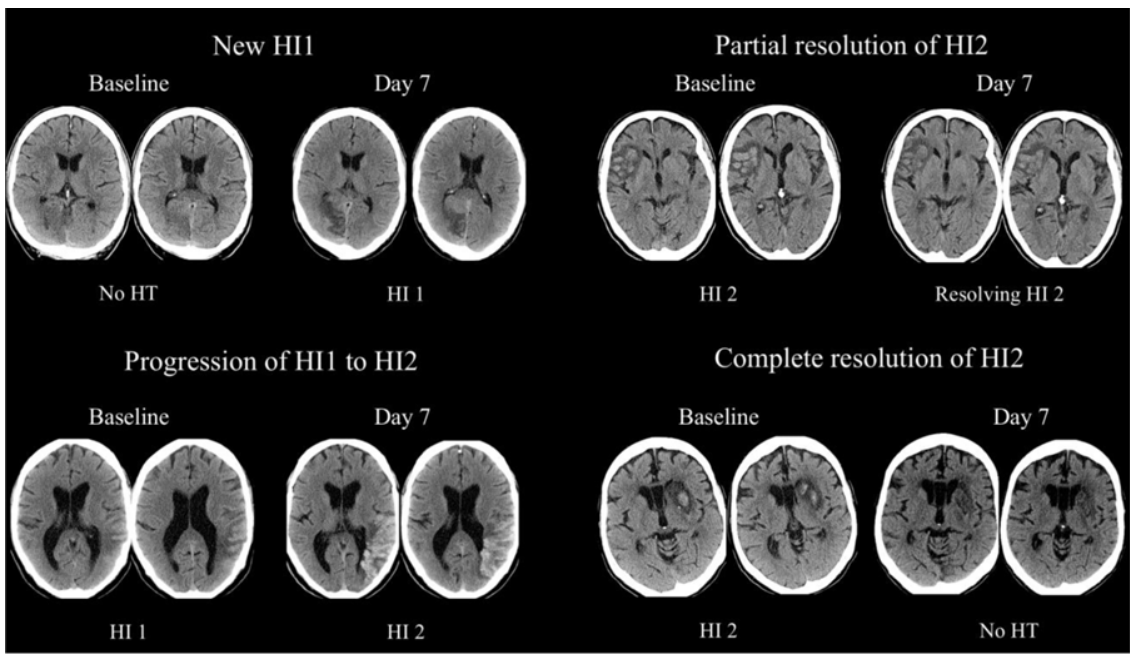

Figure 3: Examples of asymptomatic incident (new/progressive) and resolving $H T$ observed after dabigatran initiation. HII was defined as small petechiae along the margins of the infarct, and hemorrhagic infarction type 2 (HI2) as confluent petechiae within the infarcted area but no space-occupying effect.

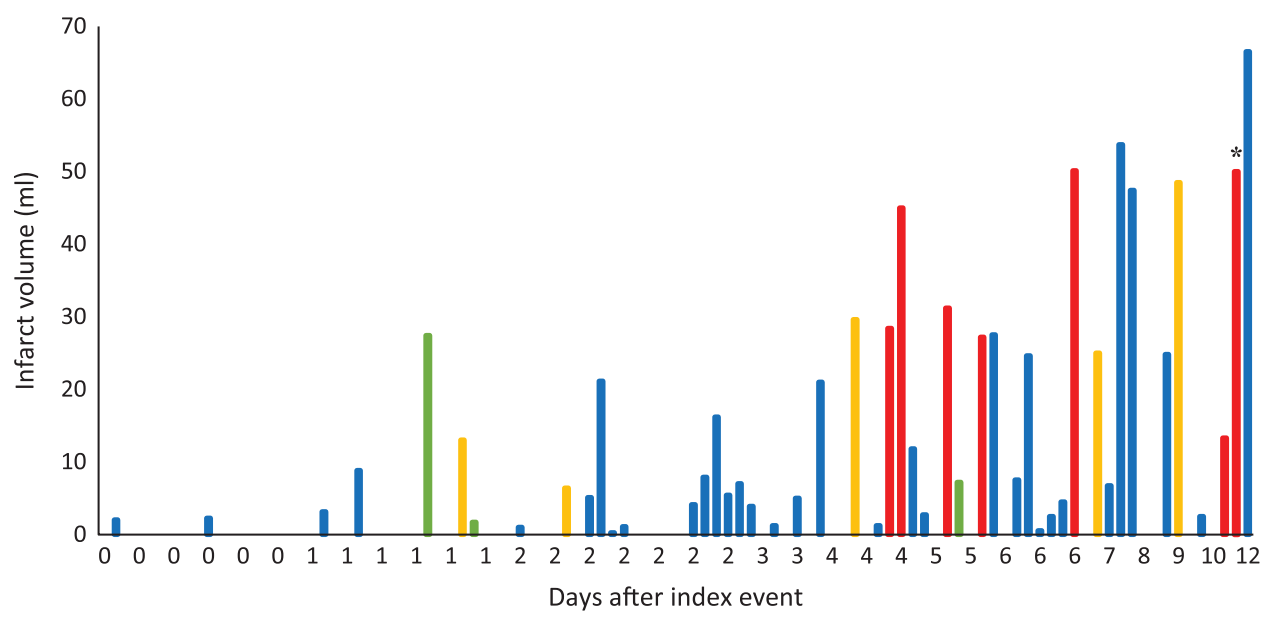

Figure 4: Relationship between baseline infarct volume and time from symptom onset to first dabigatran dose. Blue bars: No HT. Red bars: Baseline HT. Yellow bars: Incident HT at day 7. Green bars: Recurrent ischemic events. *Patient with baseline HII that progressed to HI2 at day 7.

\section{Baseline HT}

On baseline CT, HT was present in seven patients (7\%). Of these, four patients had HI1 and three patients had HI2 (Table 1). In three of these seven patients, the petechial hemorrhage completely resolved by day 7 , despite treatment with dabigatran (Figure 3). In three patients, the HT grade did not change at day 7. A single patient developed asymptomatic progression from HI1 to HI2 (Figure 3).

Median (IQR) infarct volume in patients with baseline HT was $31.2(27.8-47.5) \mathrm{ml}$, which was larger than patients without HT $0(0-5.1) \mathrm{ml}(p<0.0001)$. Dabigatran initiation in patients with baseline HT was delayed to 5 (4.5-9) days, which was significantly longer than in patients without baseline HT (2 (1) days, $p<0.0001$; Table 1). The relationship between infarct volume, baseline and incident HT, recurrent ischemic events, and time to dabigatran initiation is illustrated in Figure 4.

\section{Incident HT}

No patients developed symptomatic HT or asymptomatic PH at any point. The total number of patients with incident HT by day 7 was $6(6 \%)$. Clinically silent, but new HI1 was seen in four patients, and a single patient developed asymptomatic progression from HI1 to HI2. Another patient developed incident asymptomatic HI2 (Figure 3). Patients with incident HT had larger median (IQR) baseline infarct volume $27.3(13-48.5) \mathrm{ml}$, than those without (HT $0(0-5.4) \mathrm{ml}, p<0.0001$; Table 1). The only predictor of incident HT was infarct volume (odds ratio $=1.063$ [1.020-1.107], $p<0.003$ ).

\section{Recurrent Ischemic Events}

Recurrent ischemic events occurred within 30 days in four patients (Table 1). One of these patients also developed fatal systemic emboli 3 days after dabigatran initiation. All ischemic 
events occurred within 10 days of dabigatran initiation. There were no predictors of recurrent ischemic stroke.

\section{Clinical Outcomes}

All six patients with incident HT were functionally independent $(\mathrm{mRS}=0-2)$ at 30 days, which was similar to the good outcome rates seen in those without HT $(90 \%, p=0.422)$. Conversely, two of the four patients with recurrent ischemic events were dead or severely disabled $(\mathrm{mRS}=5)$ by day 30 . There were no systemic bleeding complications or other SAEs were reported within the study period.

\section{Discussion}

These data support the safety of initiating dabigatran in AF patients early after TIA or minor ischemic stroke. Even in cases where HT was present prior to anticoagulation, dabigatran did not result in symptomatic HT. In contrast to HT, which was always clinically silent, early recurrent ischemic events were always clinically evident and resulted in clinical disability/death in half the cases. Recurrent ischemic events occurred within 10 days of the index event, suggesting there is a cost to delaying anticoagulant therapy.

There are limited published data related to the safety of early anticoagulation with dabigatran after cardioembolic stroke or TIA. There are several retrospective analyses of AF management in acute stroke that describe early anticoagulation, primarily with heparin, and/or warfarin. ${ }^{3-6} \mathrm{~A}$ meta-analysis of heparin/lowmolecular-weight heparin/heparinoids suggested that early anticoagulation with these agents in cardioembolic stroke is harmful, due to increased rates of symptomatic HT. ${ }^{3}$ Conversely, a more recent meta-analysis suggested that recurrent ischemic stroke is more common than symptomatic HT within 90 days and that early antithrombotic therapy (antiplatelets and anticoagulants were combined) may be beneficial. ${ }^{14}$

The DOACs have all been shown to safer than warfarin with respect to intracranial hemorrhage in patients with chronic $\mathrm{AF}^{15}$ The DOACs specifically target single steps in the coagulation cascade, whereas warfarin impairs production of multiple profactors. This may theoretically make DOACs safer in the acute stroke setting but given the multifactorial and incompletely understood pathogenesis of HT, this is highly speculative.

Four prospective observational studies of early anticoagulation after stroke, included some patients initiated on an DOAC. The Stroke Acute Management with Urgent Risk-factor Assessment and Improvement study was a prospective observational study of anticoagulant decision-making in patients admitted to hospital with stroke/TIA and AF. ${ }^{16} \mathrm{~A}$ total of 1116 patients were started on warfarin $(n=650$, median 3 days) or an DOAC ( $n=466$, median 4 days) after stroke/TIA onset. Although there were no reports of intracerebral hemorrhage prior to hospital discharge, systematic neuroimaging after anticoagulation initiation was not performed. At 90 days, the recurrent stroke/systemic embolism rate was $2.84 \%$ and the rate of major bleeding was $1.1 \%$ in the DOAC treated patients. ${ }^{17}$ The early Recurrence and cerebral bleeding in patients with Acute ischemic stroke and atrial Fibrillation (RAF) study was a similar prospective observational study performed in 1092 patients that included 93 patients treated with an DOAC. ${ }^{18}$ Timing of anticoagulation was at the discretion of the treating physician, varying from 1 to 90 days, and $24 \%$ of patients were never anticoagulated. The recurrent ischemic stroke rate was $7.6 \%$ risk, and symptomatic cerebral hemorrhage occurred in 3.6\% of patients by day 90 . Analysis of data from the Clinical Relevance Of Microbleeds In Stroke-2 study assessed the effect of oral anticoagulant timing in patients with $\mathrm{AF}$ and stroke. ${ }^{19}$ The time to treatment was determined by the investigators and retrospectively dichotomized into early (0-4 days) and late ( $\geq 5$ days or never started) periods. Of 1355 patients prescribed an oral anticoagulant, 358 patients $(26 \%)$ were started early and 997 (74\%) were started late. Recurrent ischemic stroke and intracranial hemorrhage rates were similar between the two groups. The majority of patients $(65 \%)$ were treated with warfarin, rather than an DOAC. Finally, one singlecenter registry of anticoagulation practice patterns in cardioembolic stroke indicated that $65 \%$ of 155 patients prescribed an DOAC after stroke was initiated within 7 days of onset and that this was not associated with increased risk of symptomatic HT or recurrent stroke. ${ }^{20}$ Once again, however, systematic imaging was not part of this registry.

Previous assessments specific to the safety of DOAC initiation in acute cardioembolic stroke have largely been limited to retrospective and small prospective studies. One single-center study reported no symptomatic HT or recurrent stroke in 41 patients treated with DOACs at a median of 2 (IQR 5) days after onset. ${ }^{21}$ Our group previously reported a prospective assessment of the safety of rivaroxaban initiation at a median of 3 (IQR 5) days after cardioembolic stroke symptom onset. ${ }^{22}$ Using serial magnetic resonance imaging (MRI) pre- and post-treatment, we determined asymptomatic petechial HT was common at baseline (25/60) and remained clinically silent despite immediate treatment with rivaroxaban.

One randomized evaluation of rivaroxaban versus warfarin within 5 days of cardioembolic stroke has been published (Triple AXEL; Acute Stroke With Xarelto to Reduce Intracranial Hemorrhage, recurrent Embolic Stroke, and hospitaLstay). ${ }^{23}$ Rivaroxaban $(n=101)$ and warfarin $(n=94)$ were associated with similar recurrent ischemic stroke and intracranial hemorrhage rates. Asymptomatic HT on MRI performed at 4 weeks was seen in $49.5 \%$ and $54.5 \%$ of patients receiving rivaroxaban and warfarin, respectively. The higher rates of HT than seen in our study were most certainly related to the higher sensitivity of MRI for petechial bleeding, rather than differences between anticoagulants. As in our study, most patients had mild stroke symptoms (a median NIHSS score of 2 in both groups).

In the present study, we observed that the infarct volume was the only predictor of asymptomatic HT after anticoagulation. In addition, clinicians delayed dabigatran initiation in patients with larger infarcts, irrespective of the presence or absence of HT. A similar pattern was observed in the RAF study, ${ }^{18}$ reflecting common clinical practice patterns and expert recommendations. ${ }^{24}$ Symptomatic HT after anticoagulation is an infrequent event, making it difficult to predict, but thrombolysis related HT as previously been associated with infarct volume. ${ }^{25}$ The practice of delaying anticoagulation in patients with large lesions due to concerns that they are more prone to symptomatic HT appears reasonable at present. The absolute risk of HT and the optimal timing in individual patients remain unknown however.

Ultimately, the risk/benefit ratio of early versus delayed anticoagulation in AF patients with stroke will remain an area of clinical equipoise until randomized trials are completed. The only randomized trial of early dabigatran versus aspirin published to 
date was completed in patients without $\mathrm{AF}^{26}$ Several trial protocols have been published or registered. ${ }^{27}$ Patients in these trials are randomized to an DOAC, initiated as early as $48 \mathrm{~h}$ and up to 4 days after onset, or delayed to 5-10 days. The primary outcome is the composite of recurrent ischemic stroke and symptomatic HT. These include the TIMING (Timing of Oral Anticoagulant Therapy in Acute Ischemic Stroke With Atrial Fibrillation: a Prospective Multicenter Registry-based Non-Inferiority Randomized Controlled Clinical Trial, NCT02961348), ${ }^{28}$ ELAN (Early Versus Late Initiation of Direct Oral Anticoagulants in Postischaemic Stroke Patients With Atrial fibrillation, NCT03148457), START (Optimal Delay Time to Initiate Anticoagulation After Ischemic Stroke in Atrial Fibrillation, NCT03031928) trials, and OPTIMAS (OPtimal TIming of Anticoagulation After Acute Ischemic Stroke, NCT03759938).

\section{Limitations}

This non-randomized study does not provide definitive evidence for the safety of early anticoagulation after cardioembolic stroke or TIA. Cardioembolic stroke is often associated with moderate to severe deficits and larger infarct volumes. Based on our study design, these patients were systematically excluded. Caution with respect to early dabigatran use in these patients is therefore still warranted. Low rates of the events, both recurrent ischemic stroke and HT, seen as a limitation.

\section{Conclusions}

Using prospective clinical and CT scans data, early anticoagulation with dabigatran after TIA and minor cardioembolic stroke is safe and is not associated with symptomatic hemorrhage. Asymptomatic HT is associated with larger baseline infarct volumes. Evidence of baseline petechial or confluent HT (HI1 or HI2) on CT scan at the time of dabigatran initiation does not appear to increase the risk of symptomatic HT after minor stroke/ TIA. Early recurrent ischemic events may be clinically more important. This observation provides reassurance that current practice patterns are safe, but conclusive evidence will require a larger sample size.

\section{FUNDING}

This study was funded by an Investigator-Initiated Study Grant from Boehringer Ingelheim.

\section{Conflicts of Interest}

Dr. MSharma reports grants from Boehringer Ingelheim, during the conduct of the study; grants and personal fees from Bayer, grants and personal fees from Bristol Myers Squibb, personal fees from Portola, outside the submitted work; Dr. KB reports grants and personal fees from Boehringer Ingleheim, during the conduct of the study, grants and personal fees from BMS-Pfizer Alliance, grants and personal fees from Bayer, grants and personal fees from Servier Canada, outside the submitted work. The other authors have no conflicts of interest to declare.

\section{STATEMENT OF AUTHORSHIP}

AA completed the image and clinical data analyses and drafted the initial manuscript. KN, DD, BB, GS, ST, MS, HK, and AS recruited patients into the study and made critical revisions of the manuscript. LS oversaw centralized image and clinical data collection, assisted with analysis and made critical revisions of the manuscript. MSharma and KB wrote the initial protocol, obtained funding from the sponsor, recruited patients into the study, and made critical revisions of the manuscript.

\section{REFERENCES}

1. Hart RG, Palacio S, Pearce LA. Atrial fibrillation, stroke, and acute antithrombotic therapy: analysis of randomized clinical trials. Stroke 2002;33(11):2722-7.

2. Sage JI, Van Uitert RL. Risk of recurrent stroke in patients with atrial fibrillation and non-valvular heart disease. Stroke 1983;14(4):537-40.

3. Paciaroni M, Agnelli G, Micheli S, Caso V. Efficacy and safety of anticoagulant treatment in acute cardioembolic stroke: a metaanalysis of randomized controlled trials. Stroke 2007;38(2): 423-30.

4. Sandercock PA, Counsell C, Kane EJ. Anticoagulants for acute ischaemic stroke. Cochrane Database Syst Rev. 2015(3): CD000024.

5. Jorth P, Trivedi U, Rumbaugh K, Whiteley M. Probing bacterial metabolism during infection using high-resolution transcriptomics. J Bacteriol. 2013;195(22):4991-8.

6. De Schryver EL, Algra A, Kappelle LJ, van Gijn J, Koudstaal PJ. Vitamin K antagonists versus antiplatelet therapy after transient ischaemic attack or minor ischaemic stroke of presumed arterial origin. Cochrane Database Syst Rev. 2012(9):CD001342.

7. Connolly SJ, Ezekowitz MD, Yusuf S, et al. Dabigatran versus warfarin in patients with atrial fibrillation. $\mathrm{N}$ Engl $\mathrm{J}$ Med. 2009;361(12):1139-51.

8. Wein T, Lindsay MP, Cote R, et al. Canadian stroke best practice recommendations: secondary prevention of stroke, sixth edition practice guidelines, update 2017. Int J Stroke. 2018;13(4):420-43.

9. Heidbuchel H, Verhamme P, Alings M, et al. EHRA practical guide on the use of new oral anticoagulants in patients with nonvalvular atrial fibrillation: executive summary. Eur Heart J. 2013;34(27):2094-106.

10. Robb RA, Hanson DP, Karwoski RA, Larson AG, Workman EL, Stacy MC. Analyze: a comprehensive, operator-interactive software package for multidimensional medical image display and analysis. Comput Med Imag Graph. 1989;13(6):433-54.

11. Fiorelli M, Bastianello S, von Kummer R, et al. Hemorrhagic transformation within 36 hours of a cerebral infarct: relationships with early clinical deterioration and 3-month outcome in the European cooperative acute stroke study I (ECASS I) cohort. Stroke 1999;30(11):2280-4.

12. Bath PM, Iddenden R, Bath FJ. Low-Molecular-Weight heparins and heparinoids in acute ischemic stroke: a meta-analysis of randomized controlled trials. Stroke 2000;31(7):1770-8.

13. Shaw JW, Johnson JA, Coons SJ. US valuation of the EQ-5D health states: development and testing of the d1 valuation model. Med Care. 2005;43(3):203-20.

14. Abdul-Rahim AH, Fulton RL, Frank B, et al. Association of improved outcome in acute ischaemic stroke patients with atrial fibrillation who receive early antithrombotic therapy: analysis from VISTA. Eur J Neurol. 2015;22(7):1048-55.

15. Lopez-Lopez JA, Sterne JAC, Thom HHZ, et al. Oral anticoagulants for prevention of stroke in atrial fibrillation: systematic review, network meta-analysis, and cost effectiveness analysis. BMJ. 2017;359:j5058.

16. Toyoda K, Arihiro S, Todo K, et al. Trends in oral anticoagulant choice for acute stroke patients with nonvalvular atrial fibrillation in japan: the SAMURAI-NVAF study. Int J Stroke. 2015; 10(6):836-42.

17. Arihiro S, Todo K, Koga M, et al. Three-month risk-benefit profile of anticoagulation after stroke with atrial fibrillation: the SAMURAINonvalvular atrial fibrillation (NVAF) study. Int J Stroke. 2016; 11(5):565-74.

18. Paciaroni M, Agnelli G, Falocci N, et al. Early recurrence and cerebral bleeding in patients with acute ischemic stroke and atrial 
fibrillation: effect of anticoagulation and its timing: the RAF study. Stroke 2015;46(8):2175-82.

19. Wilson D, Ambler G, Banerjee G, et al. Early versus late anticoagulation for ischaemic stroke associated with atrial fibrillation: multicentre cohort study. J Neurol Neurosurg Psychiatry 2019;90(3):320-5.

20. Seiffge DJ, Traenka C, Polymeris A, et al. Early start of DOAC after ischemic stroke: risk of intracranial hemorrhage and recurrent events. Neurology 2016;87(18):1856-62.

21. Shibazaki K, Kimura K, Aoki J, Saji N, Sakai K. Early initiation of new oral anticoagulants in acute stroke and TIA patients with nonvalvular atrial fibrillation. J Neurol Sci. 2013;331(1-2): 90-3.

22. Gioia LC, Kate M, Sivakumar L, et al. Early rivaroxaban use after cardioembolic stroke may not result in hemorrhagic transformation: a prospective magnetic resonance imaging study. Stroke 2016;47(7):1917-9.

23. Hong KS, Kwon SU, Lee SH, et al. Rivaroxaban vs warfarin sodium in the ultra-early period after atrial fibrillation-related mild ischemic stroke: a randomized clinical trial. JAMA Neurol. 2017; 74(10):1206-15.

24. Diener HC, Aisenberg J, Ansell J, et al. Choosing a particular oral anticoagulant and dose for stroke prevention in individual patients with non-valvular atrial fibrillation: Part 2. Eur Heart J. 2017; 38(12):860-8.

25. Butcher K, Christensen S, Parsons M, et al. Postthrombolysis blood pressure elevation is associated with hemorrhagic transformation. Stroke 2010;41(1):72-7.

26. Butcher KS, $\mathrm{Ng} \mathrm{K}$, Sheridan P, et al. Dabigatran treatment of acute noncardioembolic ischemic stroke. Stroke 2020;51(4): 1190-8.

27. Seiffge DJ, Werring DJ, Paciaroni M, et al. Timing of anticoagulation after recent ischaemic stroke in patients with atrial fibrillation. Lancet Neurol. 2019;18(1):117-26.

28. Asberg S, Hijazi Z, Norrving B, Terent A, Ohagen P, Oldgren J. Timing of oral anticoagulant therapy in acute ischemic stroke with atrial fibrillation: study protocol for a registry-based randomised controlled trial. Trials. 2017;18(1):581. 